\title{
Hospitalización del lactante
}

\author{
Estadística del Servicio de Lactantes del Hospital Arriarán: Ensayo de clasificación. \\ Publicado en Revista Chilena de Pediatría 1938: 1000-1004
}

\author{
Autor: ARTURO BAEZA GOÑI \\ Comentario: LUISA SCHONHAUT B. ${ }^{1}$ \\ 1. Departamento de Pediatría, Facultad de Medicina Clínica Alemana - Universidad del Desarrollo.
}

Key words: Hospitalization, pediatrics, score, pediatric history.

Palabras clave: Hospitalización, pediatría, score, historia pediatría.

\section{Contexto histórico}

Los hospitales, tal como lo sugiere su nombre, son instituciones que originalmente tenían el propósito de hospedar (del latín hospes) y albergar (también del latín hospitum) a ancianos, huérfanos, moribundos y desamparados. En sus comienzos eran atendidos por voluntarios con una finalidad caritativa, mientras que la misión curativa apareció más tardíamente en la historia de estas instituciones. Es así como los primeros servicios y cátedras de pediatría se establecieron en las Casas de Menores, lugar donde llegaban los niños huérfanos $\mathrm{y}$ abandonados.

Los hospitales para niños se fundaron a principios del siglo XIX en las principales ciudades de Europa, siendo el primero de ellos el "Hopital des Enfants Malades" en París, 1802. En nuestro país los acontecimientos fueron más tardíos, aunque ya desde la segunda mitad del siglo XIX médicos y filántropos conside- raron la importancia de tener un hospital especializado en niños ${ }^{1}$.

Hacia el año 1900, Santiago tenía 2200 camas para adultos y una única sala destinada a la atención de pacientes pediátricos de 6 a 12 años, en el Hospital San Juan de Dios². Dado que esta sala no reunía las condiciones mínimas para realizar docencia, la cátedra de pediatría se desarrollaba en la Casa Nacional del Niño. A pesar que los indicadores de salud infantil eran dramáticos, fue la elevada mortalidad provocada por la epidemia de sarampión que azotó a la nación entre los años 1899-1901, seguida de otras epidemias de coqueluche y difteria, lo que finalmente sensibilizó las autoridades y motivó la fundación del primer Hospital de niños, el Roberto de Río el año $1902^{3}$.

Durante los primeros años de funcionamiento de los dispensarios pediátricos las tasas de mortalidad intrahospitalaria eran elevadísimas debido a que el financiamiento, otorgado generalmente por filántropos, escaseaba y los recursos profesionales necesarios para el adecuado cuidado de los niños, incluido la "leche de mujer" eran limitados. Por otro lado, los incipientes conocimientos científicos y arsenal terapéuticos eran poco efectivos, por 
lo que la atención "era absolutamente empírica y rudimentaria". Para el correcto análisis de la mortalidad intrahospitalaria se proponía considerar además otros factores, como "la calidad del lactante hospitalizado, en cuanto a la enfermedad que le traía al servicio, su estado nutritivo, la mayor o menor oportunidad en que recurría al médico, en fin la gravedad mayor o menor que presentaba." (Baeza Goñi A, 1938).

En el presente artículo, publicado en el año 1938, Arturo Baeza Goñi, entonces Jefe del Servicio de Lactantes del hospital Manuel Arriarán, hizo un intento de evaluar la eficiencia del hospital, para lo cual midió la mortalidad asociada a indicadores clínicos de gravedad al ingreso y patología de base. Concluyendo que "el lactante hospitalizado disfruta, en la mayor parte de los servicios modernos, de un maximum de probabilidades de mejoría, donde la mortalidad es racional y proporcionada a la calidad de los enfermos asistidos".

\section{Artículo}

La atención del lactante enfermo en servicios hospitalarios ha sido materia debatida en distintos congresos nacionales e internacionales y motivo de numerosos estudios.

En realidad, si contemplamos el camino recorrido a través de los años, comprobaremos grados de perfeccionamiento sucesivos que van dejando cada vez más atrás las grandes mortandades que se producían en los antiguos hospitales de niños, donde la atención del niño en general era absolutamente empírica y rudimentaria, para asistir hoy día a una era en que el lactante hospitalizado disfruta, en la mayor parte de los servicios modernos, de un maximum de probabilidades de mejoría, donde la mortalidad es racional y proporcionada a la calidad de los enfermos asistidos.

Sin embargo, aun no puede decirse que se haya llegado a un desideratum al respecto y de ahí que seguiremos sosteniendo que siempre es más recomendable una amplia y bien concebida y sobre todo bien controlada atención externa en Consultorios bien montados, con experto y bien organizado servicio domiciliario, que la hospitalización sin restricciones, considerando que esta debe ser estrictamente limitada a aquellos casos que, ya sea por la gravedad que revisten o por la incapacidad manifiesta de la madre o la deficiencia del hogar, lo exijan en forma perentoria.

En nuestro país, pese a nuestra alta morbilidad y mortalidad infantil, estamos muy lejos aún de poseer buenos servicios de hospitalización del lactante, principalmente por razones de orden económico. Mucho, es cierto, hemos progresado al respecto, pero falta aun muchísimo por hacer para quedar al nivel de nuestras altas necesidades.

Es el objeto de este trabajo precisamente estudiar las características que posee uno de nuestros servicios, el cual ha estado a nuestro cargo durante dos años, en un esfuerzo por analizar en la mejor manera posible sus resultados y poder así señalar el grado de eficiencia que posee para facilitar la mejor manera de perfeccionarlo.

$\mathrm{Si}$ bien las estadísticas suelen adolecer de defectos manifiestos es, sin duda, la mejor manera de conocer los resultados finales de una labor continuada y facilitar por medio de la comparación con resultados semejantes en otros medios y en otras épocas el progreso o el retroceso conseguido.

Damos a continuación la estadística global del Servicio de Lactante del Hospital Manuel Arriaran después de un año de trabajo: (1937).

\section{Número de niños atendidos:}

- 772 (menores de 1 año, 673; mayores 99).

- Altas: 432 (56\%).

- Fallecidos: 250 (32\%) antes de 48 horas: 66. Mortalidad (23,8\%),

Trasladados: 36 (5\%) (por infecciones intercurrentes).

- Existencia en $1^{\circ}$ de enero de 1938: 54 (7\%).

\section{Análisis de los dados de alta y de los fallecidos:}

a) Altas: 432 .

Estado del enfermo al salir:

$\left.\begin{array}{lrc}\text { Sano ................... } & 189 & (43,4 \%) \\ \text { Mejor ................. } & 176 & (40,7 \%)\end{array}\right\}$


b) Fallecidos: 250 .

Antes de las 24 horas ............ 53

Antes de las 48 horas ............ 13

Total ....................................66 66

Si restamos estos 66 fallecidos, sobre los cuales no podemos responsabilizar al Servicio y si, a su vez, le agregamos a la cifra que nos queda (184), los 48 niños que han sido retirados del servicio en estado peor y que seguramente han fallecido, nos quedara una cifra de 232 fallecidos, es decir, una cifra de Mortalidad verdadera de $30 \%$.

Estos datos estadísticos así presentados, no permiten en realidad dar a conocer la verdadera situación observada durante el año. No aparece reflejado un factor de importancia capital para apreciarla, cual es la calidad del lactante hospitalizado, en cuanto a la enfermedad que le traía al servicio, su estado nutritivo, la mayor o menor oportunidad en que recurría al médico, en fin la gravedad mayor o menor que presentaba y por último, los recursos con que contábamos para tratarlo como ser: personal de enfermeras diurnas y nocturnas, suficiente cantidad de leche de mujer, etc.

Es indudable que para establecer sobre bases completamente seguras estos factores, el sistema perfecto seria estudiar cada caso en particular, su edad, peso, antecedentes, diagnostico, etc. y de ellos extraer, al través de múltiples cuadros, conclusiones parciales y generales, y aun así nos quedarían algunos factores subjetivos casi imponderables, imposibles de señalar en forma objetiva.

Como este procedimiento, si exacto es engorroso y difícil, discurrimos nosotros un sistema, empírico, sin duda, y susceptible de objeciones, pero que estimamos de gran sencillez para obtener resultados prácticos en forma lo más objetivo posible. Se basa en el criterio clínico y en la experiencia adquirida por el pediatra a través de años de atender niños enfermos, no aplicado en forma exclusivamente personal, sino después de discusión y consultas de los diversos médicos del servicio. Los resultados que expondremos más adelante, creemos que nos dan razón al juzgar de utilidad práctica el sistema que exponemos a continuación.

Clasificamos según el criterio ya señalado a cada lactante hospitalizado, a más tardar al $2^{\circ}$ o $3^{\text {er }}$ día de su ingreso, en 3 grupos, a saber:

Grupo I. Aquellos niños que ingresaban en tales condiciones generales satisfactorias, con afecciones de por si benignas que nos hacían afirmar que ellas debían curar en un plazo más o menos corto. A nuestro juicio, si estos niños no sanaban completamente, significaba ello una evidente y manifiesta deficiencia del servicio. Ejemplos: Eutrofia-Gripe, Eutrofia-Sarna, Eutrofia-Dispepsia, etc.

Grupo II. Formado por niños que en condiciones inferiores a los del grupo anterior ingresaban por afecciones de mayor gravedad y de pronóstico en todo caso reservado, pero que la clínica y la experiencia nos permitían afirmar que aun cuando su estado era grave, bien atendidos podían sanar. Eran, pues, niños que sometían verdaderamente a prueba el criterio médico, la atención del personal de enfermeras, el funcionamiento de la Cocina de Dietas, etc, todo lo cual debía converger en forma satisfactoria hacia obtener la curación de estos enfermos en un tiempo más o menos largo. Ejemplo: Distrofia leve-dispepsia aguda. Distrofiabronconeumonia, Eutrofia-Toxicosis, Distrofia leve-enterocolitis, etc.

Grupo III. Lo forman aquellos niños que ingresan en tan malas condiciones generales, con enfermedades graves, algunas incurables y después de varios días de evolución y sobre los cuales el servicio en ningún caso podría hacerse responsable de su muerte más o menos rápida y que en cambio, si lograba salvarles, este hecho constituía un verdadero record de eficiencia. Estos casos, todos los conocemos y generalmente forman una cuota elevadísima de la mortalidad del lactante hospitalizado. Todo cuanto se haga por individualizarlos $\mathrm{y}$ hacer su profilaxis, resultara evidentemente en una disminución notoria de nuestra alta cifra de mortalidad en los servicios de lactantes.

Esta clasificación, lo repetimos empírica, tiene, a nuestro juicio, verdadero valor cuando se realiza por un mismo personal médico experimentado, siguiendo estrictamente las normas ya señaladas, a más tardar al segundo o tercer día de ingreso, previa discusión detenida del 
diagnóstico. Al cabo de poco tiempo de practicarla se observa una marcada uniformidad de criterio, lo cual se verá, por lo demás, bien reflejada en los resultados que expondremos a continuación.

Otro aspecto, que creemos de interés hacer resaltar, es el carácter educativo que ejerce sobre el personal, principalmente sobre las enfermeras al explicárseles claramente el valor de cada grupo y así, en poco tiempo todo el personal de nuestro servicio dejaba de pensar en el enfermo como un simple caso clínico de mayor o menor interés para recordar que estábamos frente a un niño enfermo, que si era tipo I, debía sanar, si tipo II, bien atendido podía sanar, y si del tipo III, su curación constituía un éxito indiscutible, consideraciones que no es raro ver olvidadas en los servicios de hospital en general:

\section{Resultados}

Clasificación según Grupos

\begin{tabular}{|llclc|}
\hline Ingresaron & Tipo I & $63(9 \%)$ & Fallecieron & $2(3,1 \%)$ \\
Ingresaron & Tipo II & $203(28 \%)$ & Fallecieron & $19(9,3 \%)$ \\
Ingresaron & Tipo III & $504(65 \%)$ & Fallecieron & $229(45,3 \%)$ \\
Total & & 772 & Total & 250 \\
\hline
\end{tabular}

De estas estadísticas fluyen conclusiones de orden práctico indiscutible, como ser: a) la pésima calidad de la mayoría de nuestros hospitalizados; b) que nuestra gran cifra de mortalidad es función de esta circunstancia desfavorable; c) que la gran mayoría de los que llegan en condiciones satisfactorias o cuyo estado permite hacer algo por ellos, mejora; d) que muchos, más de la mitad de aquellos niños que llegan en condiciones deplorables, a pesar de ello, han podido ser dados de alta sanos o mejores, por lo tanto, la eficiencia de nuestro servicio de lactantes es satisfactorio, y e) que es urgente hacer propaganda para que las madres recurran al hospital en forma temprana y oportuna, ya que así podemos darles la casi seguridad de salvar a sus hijos y que es urgente también ampliar los servicios de consulta externa, dotándolos de un buen servicio de atención domiciliaria, para así disminuir las hospitalizaciones tardías y acortar las estadas dentro del servicio, mediante un estricto y bien coordinado control a domicilio.

\section{Comentario}

El Hospital Manuel Arriarán fue inaugurado en 1913, inicialmente contaba sólo con atención ambulatoria, pero debido a las necesidades imperantes, seis años después, comenzó la hospitalización pediátrica ${ }^{4}$. A principios de los años 40, el Servicio de Lactantes, cuya "instalación aún era provisoria, careciendo de boxes individuales, salas de aislamiento e instalaciones higiénicas indispensables hoy día para la atención de esta clase de niños", tenía una capacidad de 53 camas atendidas por un médico jefe, 2 ayudantes y 3 médicos ad-honorem, además de una enfermera jefe y ocho enfermeras ayudantes en el día, quedando 3 en la noche (Baeza Goñi A, $1944)^{5}$.

Con la intensión de evaluar la eficacia del Servicio, Baeza Goñi construyó un score de riesgo de mortalidad, dividiendo los ingresos en 3 grupos de acuerdo a criterios clínicos de gravedad y la patología de base. En la tabla 1 se muestra una análisis comparativo de la mortalidad entre los años 1937 y $1943^{5}$.

Al grupo I pertenecían los niños eutróficos hospitalizados por cuadro leves, como gripe, sarna o dispepsia, la mortalidad de dichos niños daba cuenta de una "falla evidente del servicio que lo ha atendido"; el año 1937 fueron 2 los fallecidos en este grupo, sin verificarse nuevos casos los años posteriores.

El grupo II quizás era el que daba mejor cuenta de la eficiencia del hospital, dado que a este grupo pertenecía todo niño que clínicamente impresionaba que "sanaría si es colocado en buenas condiciones de alimentación y sometido a un tratamiento adecuado". Este fue el grupo en que se logró el más destacado el descenso de la mortalidad, desde 9,3\% el año 1937, a un solo caso en los años siguientes.

Al grupo III pertenecían los niños que se hospitalizaban en un estado de gravedad tal, que en su mayoría fallecía previo a la instalación de los apropiados cuidados médicos; "Sobre un niño de tan mala calidad, el servicio no puede ser responsable del fracaso que se ve casi seguro y, en cambio, si llega a sanar, el éxito revelaría un verdadero record de atención.”. Por lo que podemos inferir el orgullo que significaba para el equipo tratante que un 
Tabla 1. Mortalidad intrahospitalaria según índice de gravedad. Hospital Manuel Arriarán, años 1937-1942-1943

\begin{tabular}{|cccccc|}
\hline Año & $\begin{array}{c}\text { Tipo I } \\
\text { Mortalidad } \mathbf{n}(\%)\end{array}$ & $\begin{array}{c}\text { Tipo II } \\
\text { Mortalidad n (\%) }\end{array}$ & $\begin{array}{c}\text { Tipo III } \\
\text { Mortalidad n (\%) }\end{array}$ & $\begin{array}{c}\text { Total } \\
\text { hospitalizados }\end{array}$ & $\begin{array}{c}\text { Mortalidad global } \\
\text { \% }\end{array}$ \\
\hline 1937 & $2 / 63(3,1)$ & $19 / 203(9,3)$ & $229 / 504(45,3)$ & 772 & 32,4 \\
1942 & $0 / 76(0)$ & $1 / 196(0,5)$ & $158 / 347(45,5)$ & 619 & 25,5 \\
\hline 1943 & $0 / 35(0)$ & $0 / 164(0)$ & $148 / 391(37,9)$ & 614 & 24,5 \\
\hline
\end{tabular}

poco más de la mitad de los niños pertenecientes a este grupo sanaba.

Baeza Goñi atribuía las mejoras de la eficiencia hospitalaria, evidenciados en las estadísticas de los años 1942- 1943, a "la labor constantemente abnegada y eficiente del personal de médicos, ayudantes y ad-honorem y muy especial a la de las enfermeras, que realizan, sin duda alguna, la labor más delicada y que constituye, a la vez, alto exponente del grado de preparación por ellos alcanzado."5 Para asegurar el adecuado aporte de leche de mujer, considerado uno de los pilares terapéuticos, junto con las transfusiones y curaciones, se creó un lactario en el hospital Manuel Arriarán "Comenzamos la labor modestísimamente en noviembre del año 1935 con un capital de $\$ 200 " 6$.

Junto con la organización del cuidado intrahospitalario, a fines de los años 30 comenzó un auge en el desarrollo de la pediatría a nivel internacional, con el ensayo de medidas terapéuticas empíricas, algunas de ellas efectivas. Es así como la introducción de quimioterápicos y antibióticos explicaría la mayor caída histórica en la tasa de mortalidad infantil, acontecida en la década de los 40.7 Para citar un ejemplo, en la era pre-antibiótica, como fue el año 1936, un tercio de los niños hospitalizados por erisipela fallecía ${ }^{8}$; por el contrario, tres años después, el año 1939 se publicaban los primeros casos de Meningitis purulenta neumococócica tratados exitosamente con sulfanilamida, siguiendo "numerosos trabajos experimentales y clínicos realizados por investigadores norteamericanos y europeos desde 1937 adelante".

A pesar de que los indicadores parecían favorables, los pediatras no se daban por satisfechos. Era llamativo que el $60 \%$ de los niños se hospitalizaban se encontraban prácticamente fuera del alcance terapéutico debido a "condi- ciones tan deficientes de su estado nutritivo y desarrollo, con antecedentes de infecciones y trastornos nutritivos repetidos, generalmente mal atendido durante varios días en su casa", había que mejorar las condiciones de salud de la población, elevando los cuidados ambulatorios y domiciliarios; para ellos Baeza Goñi proponía "hacer propaganda para que las madres recurran al hospital en forma temprana y oportuna, ya que así podemos darles la casi seguridad de salvar a sus hijos". Estas medidas fueron implementadas exitosamente por la Pediatría Social Chilena, y sin duda fueron las principales responsables de dar un vuelco a la historia de la infancia en nuestro país.

\section{Referencias}

1.- Rojas Flores J: Historia de la infancia en el Chile republicano, 1810-2010. World color, Chile 2010.

2.- Vargas $N$ : Historia de la pediatría chilena: crónica de una alegría. Editorial Universitaria. Santiago de Chile 2002.

3.- Laval E: La epidemia de sarampión de 1899-1900 en Chile y la creación del primer hospital de niños de Santiago. Rev Chil Infectol 2002; 19 (2): 121-3.

4.- Laval E: Recuerdo histórico del antiguo Servicio de Infecciosos del Hospital de Niños Manuel Arriarán. Rev Chil Infectol 2008; 25 (4): 301-6.

5.- Baeza Goñi A: Comentarios sobre la estadística del servicio de lactantes del hospital Manuel Arriarán. Rev Chil Pediatr 1944; 15 (4): 290-6.

6.- Baeza Goñi A: EL Lactario del Hospital Manuel Arriarán. Rev Chil Pediatr 1937; 277-81.

7.- Kaempffer A, Medina E: Mortalidad infantil reciente en Chile: éxitos y desafíos. Rev Chil Pediatr 2006; 77 (5): 492-500.

8.- De la Maza V: La erisipela en la edad infantil (Revisión de 70 observaciones). Rev Chil Pediatr 1936; 394-402.

9.- Fabres $M$ : El tratamiento de la meningitis purulenta con la Sulfanilamida. Rev Chil Pediatr 1939; 533-43. 